\author{
Heiner Gabele
}

\section{Einführung}

Essen ist mehr als nur das Stillen von Hunger. Die Nahrungsaufnahme ist hochemotional besetzt und frühere Esserfahrungen und Gewohnheiten steuern zentral, was wir essen. Deswegen ist es für den Arzt nicht einfach, konstruktiv mit dem Patienten über dieses Thema zu sprechen. Gerade sog. gesunde Nahrungsmittel, wie das Vollkornbrot oder die Milch, können zu Symptomen und Krankheiten führen, die auf den ersten Blick nicht mit Nahrungsmitteln in Zusammenhang stehen. Das Themengebiet der Nahrungsmittelunverträglichkeiten ist sehr umfangreich. In diesem Artikel wird vor allem auf die nicht immunogen bedingten Nahrungsmittelintoleranzen eingegangen; die immunogen vermittelten werden gestreift. Es wird nicht auf die strukturell bedingten Intoleranzen, wie z.B. das Kurzdarmsyndrom, und die Differenzialdiagnose der Nahrungsmittelintoleranzen, wie z.B. hormonaktive Tumoren, eingegangen.

\section{Diagnostisches Vorgehen an der Aeskulap-Klinik bei Verdacht auf Nahrungsmittelintoleranz}

Bei einer schweren chronischen Erkrankung und dem Verdacht auf nahrungsmittelassoziierte Symptome gehen wir folgendermassen vor: Bei der Erhebung der Anamnese sind wir bestrebt, mögliche Zusammenhänge zwischen Nahrungsaufnahme und wiederholten körperlichen Reaktionen/Symptomen festzustellen. Unsere individuelle Diagnostik richtet sich

\title{
Nahrungsmittelunverträglichkeit ganzheitlich betrachtet
}

nach der Art und Schwere der Erkrankung. Neben der klassischen schulischen Abklärung führen wir eine Regulationsthermographie nach Prof. Rost durch, um einen Überblick über die Temperaturregulation speziell über den Head'schen Zonen der Organe zu gewinnen. Bei Nahrungsmittelunverträglichkeiten sehen wir gewöhnlich sehr warme Colon- und Leberzonen in Verbindung mit einem sehr kalten Dünndarmareal [1]. Der Uricolortest gibt uns weitere Aufschlüsse (Abb. 1) [2]. Gibt es Hinweise für eine Darmmikroökologiestörung, findet sich gewöhnlich ein typischer Farbring nach Überschichtung des Urins mit Salpetersäure. Eine Stuhluntersuchung wird angeschlossen. Sie zeigt eine deutliche Verminderung der Protektivflora, eine Vermehrung von Fäulniskeimen und eventuell eine zusätzliche Candidabelastung an. Häufig finden wir ein erniedrigtes sekretorisches Immunglobulin (Ig) A als Ausdruck einer verminderten Abwehrleistung der Darmschleimhaut. Der Entzündungsmarker Calprotectin ist erhöht, und es findet sich auch ein erhöhtes Alpha-1-Antitrypsin als Hinweis auf eine Barrierestörung der Darmmukosa. Neben diesen eher unspezifischen Stuhluntersuchungen veranlassen wir je nach Klinik die Messung der Diaminoxidase im Serum und des Gesamt-IgG auf 180 verschiedene Nahrungsmittel. Je nach Verdacht schliessen wir den Wasserstoffatemtest nach Verabreichung von Lactose oder Fructose an (Tab. 1). Bei Verdacht auf eine echte allergische Reaktion arbeiten wir mit einem Allergologen zusammen, der die IgE-vermittelten Reaktionen weiter abklärt. Die Ernährungsberaterin erarbeitet nach Vorgabe durch den Arzt zusammen mit dem Patienten ein Konzept zur Umsetzung (Rezepte, Listen von unverträglichen Nahrungsmitteln usw.).

Eine intakte und immunologisch aktive Darmmukosabarriere ist entscheidend für die Gesundheit des Menschen. Diese Barriere umfasst eine luminale, mukosale und humorale Ebene. Hier wirken in einem komplexen Zusammenspiel verschiedene Faktoren wie sekretorisches IgA, die Bakterienflora als Protektivflora mit ihren Schutzfunktionen, Verdauungsenzyme, die Schleimschicht, Gallensalze und $\mathrm{pH}$-Wertextreme hochwirksam zusammen, um die Immunogenität der Antigene und den Antigeneinstrom $\mathrm{zu}$ reduzieren. $\mathrm{Zu}$ sätzlich schützen die angeborenen

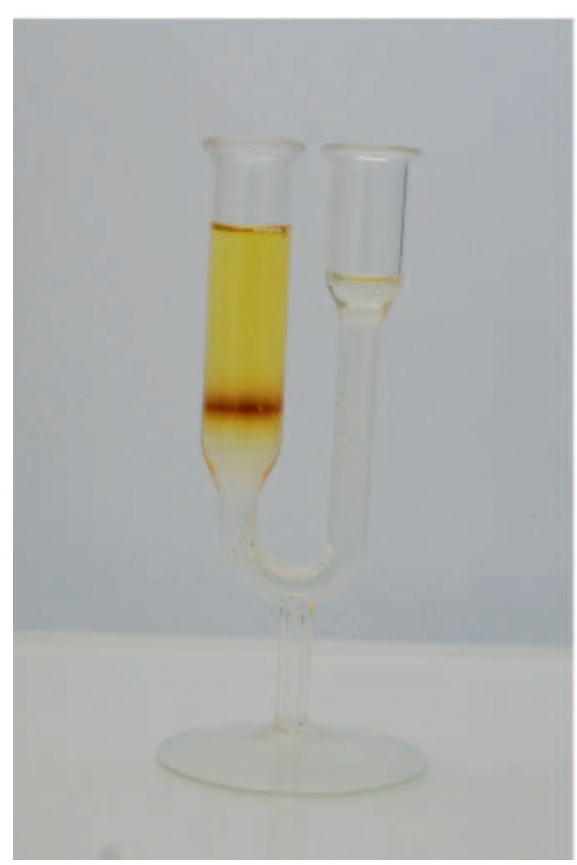

Abb.1. Uricolortest.

\section{KARGER}

Fax +497614520714 Information@Karger.d www.karger.com (c) 2011 S. Karger GmbH, Freiburg

Accessible online at: www.karger.com/szg
Dr. med. Heiner Gabele

Leitender Oberarzt Chronische Erkrankungen

Aeskulap-Klinik, Zentrum für Ärztliche Ganzheitsmedizin

6440 Brunnen am Vierwaldstättersee, Schweiz

heiner.gabele@aeskulap.com 
und erworbenen Immunfunktionen gegen Fremdantigene.

Wenn die gestörte Barrierefunktion durch die erhobenen Befunde offensichtlich geworden ist, sollte eine Restauration mit einem ganzheitlichen Konzept erfolgen [3]. Neben der Karenz der unverträglich reagierenden Nahrungsmittel ist der Wiederherstellung eines gesunden Darmmilieus die oberste Priorität zu geben. Therapeutische Massnahmen beinhalten je nach individueller Ausprägung und Schwere der Erkrankung Probiotika, Colonhydrotherapien, Phytotherapien, Substanzen aus der orthomolekularen Medizin und Neuraltherapie.

Ein von der European Academy of Allergology and Clinical Immunology (EACCI) erstelltes Flussdiagramm bietet eine systematische Übersicht über die möglichen Ursachen von
Lebensmittelunverträglichkeiten (Abb. 2). Nach der zahlenmässigen Häufigkeit soll im Folgenden auf die funktionellen Nahrungsmittelunverträglichkeiten eingegangen werden.

\section{Kohlenhydratunverträglichkeiten}

Die Kohlenhydratintoleranzen spielen quantitativ die grösste Rolle.

\section{Lactoseintoleranz}

Die Lactose, ein Disaccharid, wird durch die Lactase in Glucose und Galactose gespalten. Fehlt dieses Enzym im Dünndarm, kommt es zu einer Lactoseintoleranz, die mit ca. $70 \%$ weltweit die am häufigsten auftretende Nahrungsmittelintoleranz ist. In unseren Breiten leiden ca. 15\% an dieser Störung. Pathophysiologisch kommt es durch einen Mangel an

Tab. 1. Untersuchungen (Auswahl)

\begin{tabular}{ll}
\hline Serumanalytik & Blutsenkungsgeschwindigkeit (BSG), C-reaktives Protein, \\
& Differentialblutbild, Gesamt-IgA, -IgE, Cholestaseparameter, \\
& Spurenelementanalytik (Cu, Zn, Fe), Transglutaminase, Antigliadin, \\
& Nahrungsmitteltest IgG, IgE vermittelt \\
Wasserstoffatemteste & Lactose, Fructose, Sorbit, Lactulose, Glucose \\
Stuhluntersuchung & Pankreaselastase, Calprotectin, Alpha-1-Antitrypsin, \\
& sekretorisches IgA, Histamin, Stuhlkulturen auf pathogene Bakterien, \\
& repräsentativer Bakterienflorastatus, Pilze, Parasiten \\
Urinteste & Uricolortest, Kryptopyrol, Methylhistamin \\
Regulationsuntersuchungen & Decoderdermographie, Regulationsthermographie nach Prof. Rost, \\
& Herzratenvariabilitätsmessung \\
\hline
\end{tabular}

Spektrum der Nahrungsmittelunverträglichkeiten (NMU)

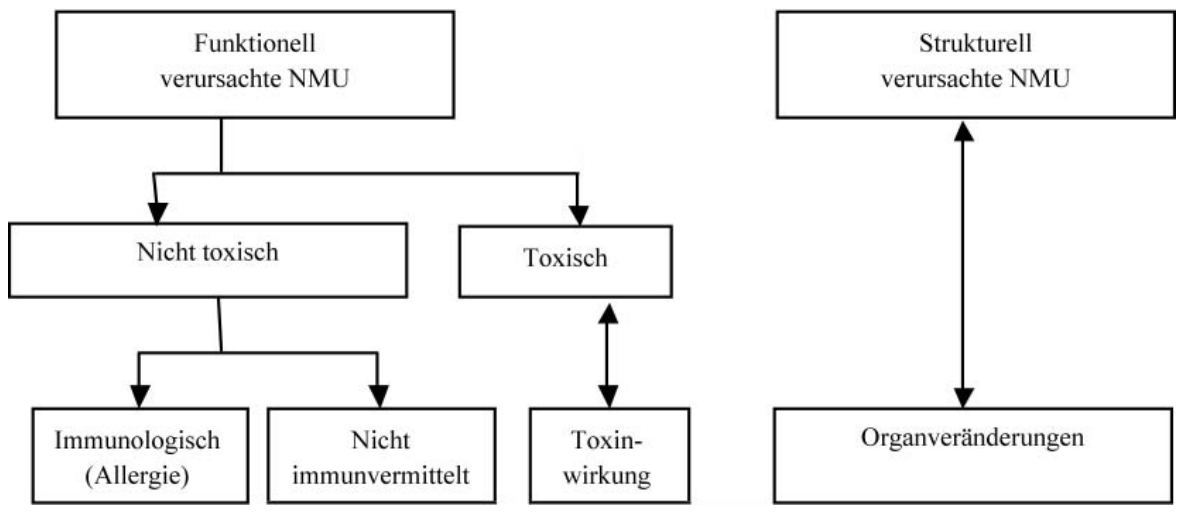

Abb. 2. Klassifikation der unerwünschten Wirkungen von Nahrungsmitteln (gemäss EACCIRichtlinien $[4,16])$.

Lactase an den Dünndarmzotten zu einer fehlenden Resorption des $\mathrm{Zu}$ ckers und konsekutiver Zersetzung durch Darmbakterien in Kohlendioxid, Wasserstoff und kurzkettige Fettsäuren mit den Folgen einer osmotischen Diarrhö, Flatulenz und Bauchkrämpfen. Man unterscheidet eine primäre Form von sekundären Formen. Die sekundären Formen entstehen durch Schädigung der Dünndarmzotten gleich welcher Ursache. Beispiele hierfür sind die Zöliakie, die bakterielle Fehlbesiedlung des Dünndarms (Small Intestinal Bacterial Overgrowth Syndrome (SIBOS)), der Morbus Crohn, das Kurzdarmsynd rom oder infektiöse Erkrankungen wie die Lambliasis. Die Diagnose der primären und der sekundären Form kann mittels des Wasserstoffatemtests gestellt werden, was als Goldstandard gilt. Nach Verabfolgung von 25-50 g Milchzucker wird alle $30 \mathrm{~min}$ die Ausatemluft auf die Wasserstoffkonzentration hin gemessen. Ein Anstieg von mehr als 20 ppm gegenüber dem Ausgangswert gilt als pathologisch (Abb. 3) [5, 6]. Ein genetischer Test ist bei der primären Form alternativ möglich, wobei hier die LCT-13910-Polymorphismen untersucht werden.

Die Therapie besteht vor allem in der Meidung von milchzuckerhaltigen Produkten, gegebenenfalls in der Verabreichung von Lactaseenzym, Calcium- und Vitamin-D-Gabe (Osteoporoseprophylaxe) und Probiotika (Verbesserung der Mikroökologie des Darms).

Fructoseintoleranz

Aufgrund einer Funktionsstörung des Transportproteins GLUT 5 in der Dünndarmschleimhaut leiden 36\% der Mitteleuropäer an einem verzögerten Fructosetransport durch den Dünndarm [7]. Von diesen leiden $50 \%$ an Symptomen wie Blähungen, Diarrhö, Bauchkrämpfen, aber auch Heisshunger auf Süssigkeiten und unter Umständen an einer depressiven Verstimmung. Die Trias Diarrhö, 
Heisshunger auf Süsses und Depression ist klinisch bedeutsam. Nicht resorbierte Fructose bildet mit Tryptophan einen Komplex und wird über den Darm ausgeschieden. Die Aminosäure Tryptophan steht als Präcursor für Serotonin ungenügend zur Verfügung. Serotonin ist beim Menschen massgeblich an der Regulation der emotionalen Stimmung beteiligt und führt bei Mangel zu einer Depression [8]. Die Diagnose wird analog zu dem Lactosetest mit der Wasserstoffgasanalyse und dem typischen Anstieg über 20 ppm nach definierter Fructosegabe $(25 \mathrm{~g})$ gestellt.

Nach verschiedenen klinischen Erfahrungen besteht bei einer Lactoseintoleranz bei bis zu 75\% der Betroffenen parallel eine Fructoseintoleranz [9]. Eine Lactoseintoleranz allein kann keine Depression auslösen, eine Fructoseintoleranz sehr wohl. Kommen jedoch beide Störungen zusammen, ergibt sich nochmals eine deutliche Verschlechterung der Depressionstiefe [10]. Einen weiteren wichtigen Einfluss spielt die Verschlechterung der GLUT-5-Transportleistung unter dem Einfluss von Zuckeralkoholen oder Zuckeraustauschstoffen wie Sorbit oder Xylit. Glucose hingegen verbessert die Transportkapazität der Fructose, idealerweise in einem Verhältnis 1:1, wie es in der Saccharose besteht. Der Fructosegehalt einzelner Nahrungsmittel kann aus einschlägigen Tabellen entnommen werden. Wenn eine typische Klinik besteht und eine Bestätigung der Fructoseintoleranz erfolgt ist, besteht die Therapie in einer Reduktion der täglichen Fruchtzuckeraufnahme und sinnvollerweise auch in einer Reduktion der Sorbitaufnahme. Zu beachten ist auch, dass Oligosaccharide wie Raffinose, Stachyose oder Verbascose, enthalten z.B. in Bohnen sowie Lauchund Kohlgemüse, die Beschwerdesymptomatik verschlechtern und deswegen initial gemieden werden sollten.

Abzugrenzen ist die sehr seltene hereditäre Fructoseintoleranz mit einer Häufigkeit von 1:130 $000 \mathrm{mit}$ einem Defekt der Fructose-1-Phosphat-Aldolase. Hierbei ist auf eine strenge Fructosekarenz zu achten.

\section{Sorbitintoleranz}

Wenn die Resorption von Sorbit vermindert ist, werden von Seiten des Darms ähnliche Symptome wie bei einer Lactose- oder Fructoseintoleranz beobachtet. Der Zuckeraustauschstoff ist in Diabetikerprodukten, in Süssigkeiten, aber auch in vielen Obstsorten (vor allem in Steinobst wie Pflaume, Aprikose, Pfirsich) vorhanden. Die Sorbitintoleranz ist bis zu $80 \%$ mit einer Fructoseintoleranz vergesellschaftet; sie kann aber auch isoliert vorkommen [11]. Der Nachweis erfolgt ebenfalls über den Wasserstoffatemtest nach definierter Sorbitgabe (5 g).

\section{Allergische Reaktionen}

Echte Nahrungsmittelallergien bestehen nicht so häufig, wie vermutet wird. Kinder leiden zu $8 \%$ und Erwachsene zu ca. $2 \%$ daran $[12,13]$. Die Mehrzahl der Nahrungsmittelallergien ist durch Kreuzreaktionen zwischen Inhalationsallergenen und Nahrungsmittelproteinen bedingt (Tab. 2) [14, 15]. Eine allergologische Diagnostik beruht auf einer exakten Nahrungsmittelanamnese unter Mitberücksichtigung eines Ernährungstagebuchs. Es wird das Gesamt-IgE bestimmt, und verdächtige allergenspezifische IgE-Antikörper werden im Serum gemessen. Hautteste auf verschiedenste Stoffe (Lebensmittelextrakte, Umweltantigene usw.) ergänzen die Untersuchungen. Als

Tab. 2. Allergene und ihre Kreuzreaktionen (Auswahl)

\begin{tabular}{|c|c|}
\hline Allergen & Häufige Kreuzreaktionen zu \\
\hline Birke & $\begin{array}{l}\text { Haselnuss, Mandel, Baumnuss, Kernobst (z.B. Apfel, Birne), Steinobst } \\
\text { (z.B. Pfirsich, Kirsche, Aprikose, Pflaume) }\end{array}$ \\
\hline Beifuss & $\begin{array}{l}\text { Sellerie, Karotte, Paprika, Sonnenblumenkerne, Erdnüsse, Kräuter und Gewürze } \\
\text { (Muskatnuss, Pfeffer, Anis, Koriander, Kümmel, Estragon, Senf, Majoran, Ingwer, } \\
\text { Thymian, Oregano, Kamille, Petersilie, Dill, Basilikum, Zimt) }\end{array}$ \\
\hline Getreide/Gräser & $\begin{array}{l}\text { Roggen, Hafer, Weizen, Gerste, Hülsenfrüchte, Pfefferminze, Kräuter/Gewürze, } \\
\text { Tomate }\end{array}$ \\
\hline Hausstaub & Krustentiere (Shrimps, Scampi, Garnelen, Langusten, Hummer, Krebse, Krabben) \\
\hline Latex & $\begin{array}{l}\text { Kartoffel, Avocado, Tomate, Banane, Kiwi, Papaya, Passionsfrucht, Pfirsich, } \\
\text { Maroni/Esskastanie, Feige, Sellerie, Buchweizenmehl, Paprika, Mango }\end{array}$ \\
\hline
\end{tabular}
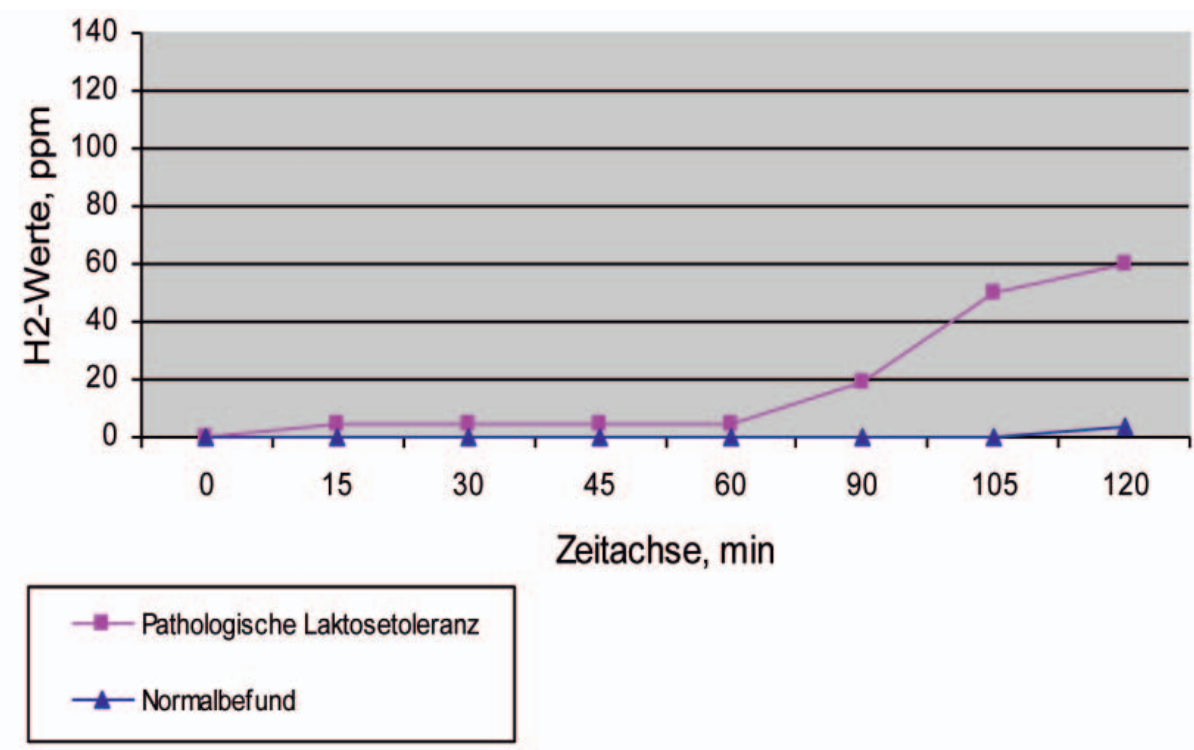

Abb. 3. Beispiel Lactoseintoleranz-/Atemtest (Wasserstoffatemtest). 
Medikamente, die die Diaminoxidase hemmen (Auswahl)

Nahrungsmittel, die vermehrt Histamin freisetzen (Histaminliberatoren)

Analgetische Medikamente, die vermehrt Histamin freisetzen (Beispiele für Histaminliberatoren)

Weitere Faktoren, die die Histaminfreisetzung fördern
Acetylcystein, Amitryptilin, Clavulansäure, Metamizol, Metoclopramid

Zitrusfrüchte, Nüsse, Weizenkeime, Alkohol

Diclofenac, Indometacin, Acetylsalicylsäure

Infektionen, Sport, seelische Erregung (Stress), chronische Erkrankungen (z.B. chronische Niereninsuffizienz)
Goldstandard gilt der Nachweis einer allergeninduzierten Reaktion am Patienten bzw. am Organsystem. Wenn damit das Krankheitsbild nicht geklärt werden kann, ist eine Provokationstestung oder eine weiterführende internistische Differenzialdiagnostik mit gezielter endoskopischer Allergiediagnostik sinnvoll [16]. Therapeutische Massnahmen bestehen in der Vermeidung der Allergieauslöser, einer homöopathisch konstitutionellen Behandlung und einer Darmmilieutherapie.

\section{Pseudoallergische Reaktionen}

\section{Salicylatunverträglichkeit}

In Europa sind bis zu 2,5\% der Bevölkerung von dieser nicht immunologisch vermittelten Aktivierung von basophilen und eosinophilen Leukozyten, Makrophagen, Mastzellen, Thrombozyten und Lymphozyten betroffen. Die Salicylate verursachen über die Zyklooxygenasehemmung eine verminderte Prostaglandinsynthese. Bei dieser Personengruppe dominieren Symptome des Respirationstrakts, die sich in Form einer Rhinosinusitis, Polyposis nasi (bis 30\% beteiligt) oder eines Asthma bronchiale (bis 10\%) äussern. Am Verdauungstrakt zeigen sich Diarrhö und Kolitis, an der Haut eine Urtikaria oder ein Quinckeödem. Nicht nur eine medikamentöse Prostaglandinsynthesehemmung kann Symptome verursachen, auch das in verschiedenen Nahrungsmitteln enthaltene Salicylat (unter anderem in Rosinen, Orange, Apfel, Curry, Paprika) muss beachtet werden. Gold- standard zur Diagnosestellung ist der Expositions-/Provokationstest. In Zweifelsfällen oder bei Kontraindikationen stehen In-vitro-Teste zur Verfügung [17].

Therapeutisch ist die Expositionskarenz entscheidend. In schweren Fällen stehen Glucocorticoide zur Verfügung. Eine Applikation der Acetylsalicylsäure in steigender Dosierung als eine Art Desaktivierung soll gute Resultate bringen [18].

\section{Histaminintoleranz}

Je nach Studie ist die Histaminintoleranz mit 1-2\% Prävalenz in der Bevölkerung keine seltene Erkrankung [19, 20]. Überwiegend sind Frauen im mittleren Alter betroffen. Die Ursache einer Histaminintoleranz kann in einer vermehrten Zufuhr histaminreicher Nahrungsmittel, einer vermehrten Freisetzung von Histamin durch Histaminliberatoren (Nahrungsmittel, Medikamente), einem eingeschränkten Abbau des Histamins durch eine verminderte Aktivität der im Darm wirksam werdenden Diaminoxidase oder einer Hemmung des Enzyms durch verschiedene Stoffe wie Alkohol und Medikamente liegen. Eine kurze Übersicht histaminreicher sowie histaminfreisetzender Lebensmittel findet sich in Tabelle 3. Symptome können Durchfall, Blähungen und Koliken seitens des Magen-DarmTrakts, nasale Kongestion, Niesanfälle und Asthma bronchiale von Seiten des Respirationstrakts sein. Es können aber auch Kreislaufwirkungen wie hypotone Zustände oder Herzrhythmusstörungen und Schmerzzustände wie Kopfschmerzen, Migräne, Dysmenorrhö und
Symptome der Haut wie Pruritus oder Urticaria beobachtet werden.

Die Diagnose wird gestellt, wenn sich mindestens zwei typische Histaminintoleranzsymptome in zeitlichem Zusammenhang mit der Aufnahme von histaminhaltigen Nahrungsmitteln und die Besserung der Symptome nach einer histaminarmen Diät zeigen [21]. Eine alleinige Bestimmung der Enzymaktivität der Diaminoxidase im Serum reicht nach einigen Autoren für die Diagnose nicht aus, da die Aktivität im Serum nicht die Aktivität des Enzyms im Darm widerspiegelt [22]. In der Aeskulap-Klinik bestimmen wir die Diaminoxidaseaktivität und eventuell auch den Histamingehalt in den Fäzes. Wenn diese sich pathologisch verändert zeigen, veranlassen wir eine zweiwöchige strenge Karenz der Nahrungsmittel, die den Histaminspiegel direkt oder indirekt erhöhen, und der entsprechenden Medikamente. Falls sich die Symptome wesentlich bessern lassen, ist die Diagnose gesichert.

Beim Schmerzpatienten ist unserer Erfahrung nach häufig eine Mitbeteiligung dieser Erkrankung zu konstatieren. Es ist auch nicht verwunderlich, da viele peripher und zentral wirksame Schmerzmittel den Histaminabbau hemmen oder die Histaminfreisetzung fördern können.

Die Therapie beinhaltet eine Verbesserung des Darmmilieus durch Colonhydrotherapie, Probiotika und Bitterdrogen, eine Verminderung der Histaminbelastung über die Nahrung und das Vermeiden histaminfreisetzender Nahrungsmittel und Medikamente. Höher dosiertes Vitamin C und oligomeres Procyanidin (OPC) 
helfen, den Histaminspiegel zu reduzieren.

Weitere biogene Amine spielen als Auslöser pseudoallergischer Reaktionen eine Rolle, wie z.B. Phenylaethylamin (unter anderem in Schokolade, Fleischextrakt) oder Tyramin (unter anderem in Orangen, Tomaten). Ursächlich beteiligt können aber auch Konservierungsstoffe wie Sulfite und Benzoesäure oder der Geschmacksverstärker Glutamat sein.

\section{Sonderform Zöliakiel}

einheimische Sprue

Die kindliche Zöliakie oder die Erwachsenenform einer manifesten Sprue sind nicht so seltene Erkrankungen, deren Häufigkeit in unseren Breiten mit ca. $1 \%$ angegeben wird. Die typischen Bauchsymptome sind maximal nur bei $30-40 \%$ der Betroffenen vorhanden. An verschiedensten Erkrankungen kann die Sprue ursächlich beteiligt sein. Am Skelett ist bei einer Osteoporose oder einer Osteomalazie daran zu denken. Außerdem kann sie hinter einer Amenorrhö, einer Infertilität oder einem sekundären Hyperparathyreoidismus stecken. Eine chronische Hepatitis, eine chronische Cholangitis, aber auch eine Dermatitis herpetiformis, ein peripheres Ödem oder eine Blutungsneigung im Sinne von Ekchymosen können darauf hinweisen. Eine Vitamin-B12- oder eine Eisenmangelanämie oder eine peripher sensible Polyneuropathie, eine Kachexie oder eine allgemeine Schwäche sind mögliche Folgen. Interessant ist auch eine überzufällige Häufigkeit der Sprue mit einem Diabetes mellitus Typ 1, einer chronisch entzündlichen Darmerkrankung, einer Hashimotothyreoiditis, einer rheumatoiden Arthritis, einer Sarkoidose, einer IgA-Nephritis, einer primär biliären Cholangitis oder einer primär sklerosierenden Cholangitis.

Die Ursache liegt in einer gestörten Immunantwort auf das Pflanzenprotein Gluten, das in verschiedenen Kulturgräsern wie Weizen, Roggen,
Triticale, Dinkel, Grünkern, Gerste, Emmer, Kamut und Einkorn vorkommt. Gluten setzt sich aus Prolaminen und Glutelinen zusammen. Die nachteilige immunologische Reaktionen auslösenden Eiweisse heissen Gliadine, die von den Prolaminen abstammen. Farrell [23] teilt eine Sprue in eine latente Form, die sich serologisch zeigen kann, eine «Silent»-Form, die auch mit einer Malabsorption einhergehen kann, und eine manifeste Sprue mit der typischen Klinik, Malabsorption, Histologie und Serologie ein. Die Diagnose wird durch die klinische Symptomatik, die nachgewiesene Malabsorption, den Antikörpernachweis (Gliadin-, Transglutaminase- und Endomysiumantikörper) und durch die Dünndarmschleimhautbiopsie gestellt. Genetisch findet man eine enge Beziehung zum HLA Genotyp DQ2 von über 90\% [24]. Therapeutisch steht die strenge Expositionskarenz glutenhaltiger Lebensmittel im Vordergrund. Die Regeneration des Dünndarmepithels dauert danach Monate, sodass auch eine Substitution von Vitaminen (z.B. Vitamin B1, B12, D), Mineralien (Calcium, Magnesium), Spurenelementen (Eisen, Zink) und eventuell Eiweiss erfolgen muss.

\section{Nahrungsmittelunverträglichkeit Typ Immunglobulin $G$}

IgG-basierte Immunreaktionen als Ursache von Nahrungsmittelunverträglichkeiten werden von den Allergologen ausser bei einer Sprueerkrankung einhellig abgelehnt [25-27]. Regelmässig verzehrte Lebensmittel produzieren eine Immunantwort in Form von IgG-Antikörpern gegen die entsprechenden Nahrungsproteine, was als normale Immunantwort gesehen wird. Tatsächlich finden sich diese IgG-Reaktionen auf Nahrungsmittel bei vielen Gesunden. Eine moderate Bildung von nahrungsspezifischen IgG-Antikörpern ist physiologisch. Unter bestimmten Voraussetzungen können diese Antigen-/Antikörperkomplexe jedoch nicht mehr genügend durch das
Phagozytosesystem eliminiert werden. Es kommt zu einer Aktivierung von Entzündungskaskaden, was je nach Ausmass auch zu klinischen Symptomen führen kann. Wenn ein Patient unter unklaren, chronischen Beschwerden leidet, die nicht durch eine klassische Diagnose erklärt werden können, muss aus unserer Sicht und Erfahrung an diese Art von Nahrungsmittelintoleranz gedacht werden. Gerade unspezifische Symptome wie Müdigkeit, Erschöpfung, wechselnde Schmerzzustände oder abdominelle Symptome sind oft sehr schwierig diagnostisch einzuordnen. Wenn der IgG-Nahrungsmitteltest dann starke Reaktionen auf bestimmte Stoffe, wie z.B. auf Ananas, zeigt und dieses Nahrungsmittel weggelassen wird, findet sich häufig eine deutliche Besserung der Symptome. Ist es eine Placebowirkung? Wir haben diesen Test an der Aeskulap-Klinik seit Jahren etabliert und schätzen seine Aussage im ganzheitlich diagnostischen Kontext. Dieses Testergebnis muss immer mit allen Befunden zusammen interpretiert werden und ist als einzelner Diagnosebaustein nicht valide. Wir benutzen die Messung der Gesamt-IgG auf 180 verschiedene Nahrungsmittel. Vor allem die Subklassen IgG1 und 3 reagieren bevorzugt mit den Nahrungsproteinen, aktivieren dabei Komplement, binden mit hoher Affinität an Fcgamma-Rezeptoren der Phagozyten und der natürlichen Killerzellen und vermitteln die antikörperinduzierte zelluläre Toxizität. Bislang gibt es nur wenige Studien zu dieser Thematik [28-31]. Nahrungsspezifische IgGAntikörpertiter definieren noch keine Krankheit. Wenn aber unerklärliche klinische Symptome vorhanden sind und die Symptome durch eine Expositionskarenz des durch den Test identifizierten Lebensmittels verschwinden, muss dies als Hinweis gelten. Wenn eine Reexposition diese Symptome wieder aufflammen lässt, ist dies ein starkes Indiz für deren ursächliche Bedeutung. 
Die Therapie besteht in einer Expositionskarenz, die von der Schwere der Reaktion im Test abhängig ist. Wie schon angesprochen wurde, führen wir immer begleitend eine Darmmilieutherapie durch [3].

\section{Psychosomatik der \\ Lebensmittelintoleranzen}

Der Blick auf die Psyche als Ursache sollte erst erfolgen, wenn die somatische Seite sauber abgeklärt wurde. Psychosomatische Lebensmittelintoleranzen entstehen oft als Lernerfahrungen über eine emotionale oder unbewusste Vermittlung [32]. Die psychosomatisch bedingte Nahrungsmittelintoleranz ist also eine Ausschlussdiagnose. Psychische Reaktionen sind aber sehr häufig auffällig mitbeteiligt; nicht ursächlich, aber als Begleitreaktion, was therapeutisch beachtet werden sollte.

\section{Patientenbeispiel}

Die Patientin - Frau M., 65 Jahre berichtete über eine seit 10 Jahren bestehende Muskelschwäche mit einer ausgeprägten Erschöpfbarkeit, die eine maximale Gehdauer von 15 min erlaubte. Verschiedenste Abklärungen bei mehreren Fachärzten inklusive einem Neurologen konnten das Phänomen weder einordnen noch erklären. Der durchgeführte Nahrungsmitteltest IgG zeigte keine wesentliche Auffälligkeit ausser einer starken Reaktion auf Vanille. Die Patientin berichtet daraufhin, dass sie seit mehr als 10 Jahren jeden Morgen Rotbuschtee mit Vanille trinken würde. Nach Absetzen des Tees kehrte umgehend die alte Kraft zurück. Nach erneutem Trinken des Tees tra- ten die alten Symptome sofort wieder auf. Diese Störung ist nie mehr aufgetreten, nachdem Frau M. bewusst auf Vanille verzichtet hatte.

\section{Literatur}

1 Rost A: Lehrbuch der Regulationsthermographie. Stuttgart, Hippokrates, 1994.

2 Herget HF: Kopf- und Gesichtsschmerz. Marburg, KVM, 2000.

-3 Ogal HP, et al: Symbioselenkung und Mikrobiologische Therapie. Eine essenzielle Basistherapie für die erfolgreiche Behandlung vieler chronischer Erkrankungen. Schweiz Z Ganzheitsmed 2011;23:33-38.

4 Bruijnzeel-Koomen C, et al: Adverse reactions to food. European Academy of Allergology and Clinical Immunology Subcommittee. Allergy 1995;50:623-635.

5 Ledochowski M: Laktoseintoleranz und Milchunverträglichkeiten. Innsbruck, Ledochowski, 2008.

6 Lomer MC, et al: Lactose intolerance in clinical practice - myths and realities. Aliment Pharmacol Ther 2008;27:93-103.

7 Ledochowski M, et al: Fructose malabsorption is associated with decreased plasma tryptophan. Scan J Gastroenterology 2001;36:367371.

8 Dills WL Jr: Protein fructosylation: fructose and the Maillard reaction. Am J Clin Nutr 1993;58(suppl 5):779S-787S.

$\checkmark 9$ Mishkin D, et al: Fructose and sorbitol malabsorption in ambulatory patients with functional dyspepsia: comparison with lactose maldigestion/malabsorption. Dig Dis Sci 1997;42: 2591-2598.

10 Ledochowski M, et al: Carbohydrate malabsorption syndromes and early signs of mental depression in females. Dig Dis Sci 2000;45:1255-1259.

11 Ledochowski $\mathrm{M}$, et al: Kohlenhydratmalabsorptionssyndrome; in Widhalm K, DialloGinstl E (Hrsg): Ernährungsmedizin. Wien, ÖÄK, 2000.

12 Cianferoni A, Spergel JM: Food allergy: review, classification and diagnosis. Allergol Int 2009;58:457-466.

13 Seitz C, et al: Nahrungsmittelallergie bei Erwachsenen - über- oder unterschätzt? Dtsch Ärzteblatt 2008;105:715-723.

14 Bonds RS, et al: A structural basis for food allergy: the role of cross-reactivity. Curr Opin Allergy Clin Immunol 2008;8:82-86.

15 Katelaris $\mathrm{CH}$ : Food allergy and oral allergy or pollen-food syndrome. Curr Opin Allergy Clin Immunol 2010;10:246-251.
16 Zopf Y, et al: Differenzialdiagnose von Nahrungsmittelunverträglichkeiten. Dtsch Ärzteblatt 2009;106:359-369.

17 Baenkler HW: Salicylatintoleranz: Pathophysiologie, klinisches Spektrum, Diagnostik und Therapie. Dtsch Ärzteblatt 2008;105:137-142.

18 Gosepath J, Schäfer D, Mann WJ: AnalgetikaIntoleranz. Langzeitergebnisse bis zu 3 Jahren bei adaptiver Desaktivierung mit einer täglichen Erhaltungsdosis von $100 \mathrm{mg}$ Aspirin. Laryngo-Rhino-Otol 2002;81:732-738.

19 Beineke M: Diaminoxidase (DAO) - der Marker zur Diagnose einer Histamin-Intoleranz. Bioscientia 2006;7:1-5.

20 Maintz L, Novak N: Histamine and histamine intolerance. Am J Clin Nutr 2007;85:11851196.

21 Jarisch R: Histamin-Intoleranz. Ärztemagazin 2004;8:1-4.

22 Töndury B, et al: Histaminintoleranz: wie sinnvoll ist die Bestimmung der DiaminoxidaseAktivität im Serum in der alltäglichen klinischen Praxis. Allergologie 2008;31:350-356.

23 Farrell RJ, Kelly CP: Celiac sprue. N Engl J Med 2002;346:180-188.

24 Barker JM, Liu E: Celiac disease: pathophysiology, clinical manifestations and associated autoimmune conditions. Adv Pediatr 2008;55:349-365.

25 Stapel SO, et al: Testing for $\operatorname{IgG}(4)$ against food is not recommended as a diagnostic tool: European Academy of Allergy and Clinical Immunology (EAACI) position paper. Allergy 2008;63:793-796.

26 Kleine-Tebbe J, et al: Nahrungsmittelallergie und -unverträglichkeit: bewährte statt nicht evaluierte Diagnostik. Dtsch Ärzteblatt 2005;102:A1965-A1969.

27 Mullin GE, et al: Testing for food reactions: the good, the bad, and the ugly. Nutr Clin Pract 2010;25:192-198.

28 Atkinson W, et al: Food elimination based on IgG antibodies in irritable bowel syndrome: a randomised controlled trial. Gut 2004;53:1459-1464.

29 Bernardi D, et al: Time to reconsider the clinical value of immunological IgG4 to foods? Clin Chem Lab Med 2008;46:687-690.

30 Zar S, et al: Food-specific serum IgG4 and IgE titers to common food antigens in irritable bowel syndrome. Am Gastroenterol 2005;100:1550-1557.

31 Matthews MC: Food sensitivity and its impact on the long-term chronically unwell. York, York Nutritional Laboratories Ltd, 2000.

- 32 King SC, Meiselmann HL: Development of a method to measure consumer emotions associated with food. Food Qual Pref 2010; 21:168-177. 